\title{
Introducing the Evolutionary Cell Memory (ECM) Hypothesis
}

\author{
Zaminpira S* $^{*}$ and Niknamian $S$ \\ Ph.D. in Cellular and Molecular Biology, University of Cambridge, United Kingdom \\ *Corresponding author: Somayeh Zaminpira, PhD, Cellular and Molecular \\ Biology, University of Cambridge, United Kingdom, Tel: +989121939806; E-mail: \\ saina.monthly@gmail.com
}

\section{Research Article \\ Volume 3 Issue 1}

Received Date: December 11, 2017

Published Date: January 08, 2018

\section{Abstract}

This research study has gone through more than 34 sample tumors in Violet Cancer Institute (VCI) to find the cancer cells' resemblances to the primitive eukaryote cells in 3.5 billion years ago before the entrance of the mitochondria into the eukaryote cells as endosymbionts. Nearly all the samples showed that the mitochondria inside the cells were not working properly. Their cristae were damaged or the mitochondria did not work or better said "shut down" inside the cancer cells. This study introduces a new hypothesis called the Evolutionary Cell Memory (ECM) based on the Lamarckian Evolutionary Hypothesis and the Evolutionary Metabolic Hypothesis of Cancer introduced by the Somayeh Zaminpira and Sorush Niknamian in 2017.

Keywords: ECM Hypothesis; Mitochondrion; Lamarckian Evolution; Cancer Cell; Eukaryotic Cell; Fermentation; Oxidative Phosphorylation

Abbreviations: VCI: Violet Cancer Institute; ECM: Evolutionary Cell Memory; ATP: Adenosine 3Phosphate; $\mathrm{CO}_{2}$ : Carbon dioxide; ROS: Reactive Oxygen Species; RNS: Reactive Nitrogen Species; NOS2: Nitric Oxide Synthase 2; EMHC: Evolutionary Metabolic Hypothesis of Cancer.

\section{Introduction}

\section{Eukaryotic Cell Respiration}

In eukaryotic cells, the respiration process goes through aerobic and anaerobic. The aerobic respiration mainly happens in mitochondrion which requires oxygen for creating Adenosine 3-Phosphate (ATP) [1]. Despite consuming protein, carbohydrates and fats as reactants, it is the preferred method of pyruvate breakdown in glycolysis and requires that pyruvate enter the mitochondria in order to be fully oxidized by the Krebs cycle. The products of this process are carbon dioxide and water, but the energy transferred is used to break bonds in ADP as the third phosphate group is added to form ATP (adenosine triphosphate), by substrate-level phosphorylation, NADH and 2FADH2 [Baily, Regina, Cellular Respiration].

The simplified equation is mentioned below [2]: $\mathrm{C}_{6} \mathrm{H}_{12} \mathrm{O}_{6}(\mathrm{~s})+6 \mathrm{O}_{2}(\mathrm{~g}) \rightarrow 6 \mathrm{CO}_{2}(\mathrm{~g})+6 \mathrm{H}_{2} \mathrm{O}(\mathrm{l})+$ heat

The potential of NADH and FADH2 is converted to more ATP through an electron transport chain with oxygen which is the terminal electron acceptor. Most of the ATP produced by aerobic cellular respiration is produced by oxidative phosphorylation. This works by the energy released in the consumption of pyruvate to create a chemiosmosis potential by pumping protons across the cell membrane. This potential is then used to drive ATP synthase and produce ATP from ADP and a phosphate group [3]. By going through the respiration process formula, 38 ATP molecules can be made per oxidized glucose molecule during cellular respiration. 


\section{International Journal of Biochemistry \& Physiology}

Which is, 2 from glycolysis, 2 from the Krebs cycle, and about 34 from the electron transport system. But, this maximum yield is never reached since the losses due to leaky membranes as well as the cost of moving pyruvate and ADP into the mitochondrial matrix, and current estimates range around 29 to 30 ATP per glucose [4].

Aerobic metabolism is almost 15 times more efficient than anaerobic metabolism which yields 2 molecules ATP per 1 molecule glucose. Glycolysis is a metabolic pathway that happens in the cytosol of cells in all living organisms [5]. This pathway can function with or without the presence of oxygen. In humans, aerobic conditions produce pyruvate and anaerobic conditions produce Lactic acid. In aerobic conditions, the process converts one molecule of glucose into two molecules of pyruvic acid, generating energy in the form of two molecules of ATP [6].

Four molecules of ATP per glucose are actually produced; however, two are consumed as part of the preparatory phase. The initial phosphorylation of glucose is required to increase the reactivity in order for the molecule to be cleaved into two pyruvate molecules by the enzyme aldolase. During the pay-off phase of glycolysis, four phosphate groups are transferred to ADP by substrate-level phosphorylation to make four ATP, and two NADH are produced when the pyruvate are oxidized [7].

Glucose $+2 \mathrm{NAD}++2 \mathrm{Pi}+2 \mathrm{ADP} \rightarrow 2$ pyruvates +2 $\mathrm{NADH}+2 \mathrm{ATP}+2 \mathrm{H}++2 \mathrm{H}_{2} \mathrm{O}+$ heat

Starting with glucose, 1 ATP is used to donate a phosphate to glucose to produce glucose 6phosphate. Glycogen can be converted into glucose 6-phosphate as well with the help of glycogen phosphorylase [8]. During energy metabolism, glucose 6-phosphate becomes fructose 6-phosphate. An additional ATP is used to phosphorylate fructose 6-phosphate into fructose 1,6-disphosphate by the help of phosphofructokinase. Fructose 1,6-diphosphate then splits into two phosphorylated molecules with three carbon chains which later degrades into pyruvate. Glycolysis can be translated as sugar splitting [9].

\section{Oxidative Phosphorylation}

In eukaryotes, oxidative phosphorylation occurs in the mitochondrial cristae. It comprises the electron transport chain that establishes a proton gradient (chemiosmosis potential) across the boundary of inner membrane by oxidizing the NADH produced from the Krebs cycle. ATP is synthesized by the ATP synthase enzyme when the chemiosmosis gradient is used to drive the phosphorylation of ADP. The electrons are finally transferred to exogenous oxygen and, with the addition of two protons, water is formed [10].

\section{Fermentation}

Without oxygen, pyruvate pyruvic acid is not metabolized. However, goes through the process of fermentation [11]. The pyruvate is not transported into the mitochondrion, but remains in the cytoplasm, where it is converted to waste products that may be removed from the cell. This serves the purpose of oxidizing the electron carriers so that they can perform glycolysis again and removing the excess pyruvate. Fermentation oxidizes NADH to NAD+ so it can be re-used in glycolysis. In the absence of oxygen, fermentation prevents the buildup of NADH in the cytoplasm and provides NAD+ for glycolysis [12]. This waste product varies depending on the organism. In skeletal muscles, the waste product is lactic acid. Fermentation is less efficient at using the energy from glucose because only 2 ATP are produced per glucose, compared to the 38 ATP per glucose theoretically produced by aerobic respiration. This is because the waste products of fermentation still contain chemical potential energy that can be released by oxidation [13].

The total ATP yield in ethanol or lactic acid fermentation is only 2 molecules coming from glycolysis, because pyruvate is not transferred to the mitochondrion and finally oxidized to the carbon dioxide $\left(\mathrm{CO}_{2}\right)$, but reduced to ethanol or lactic acid in the cytoplasm [14].

\section{Lactic Acid Fermentation}

Lactic acid fermentation is a metabolic process in which glucose is converted to cellular energy and the metabolite lactate. It is an anaerobic fermentation reaction that occurs in some animal cells, such as muscle cells [15-17].

If oxygen is present in the cell, many organisms will undergo cellular respiration. Hence, facultative anaerobic organisms will both ferment and undergo respiration in the presence of oxygen. Sometimes even when oxygen is present and aerobic metabolism is happening in the mitochondria, if pyruvate is building up faster than it can be metabolized, the fermentation will happen anyway. Lactate dehydrogenase catalyzes the interconversion of pyruvate and lactate with concomitant interconversion of NADH and NAD+ [18].

\section{Oxidative Damage}

In aerobic organisms, the energy needed for biological functions is produced in the mitochondria via the electron transport chain [19]. In addition to energy, reactive oxygen species (ROS) with the potential to cause cellular damage are produced. ROS can damage DNA, RNA, proteins and polyunsaturated fats in the cell membrane [20]. ROS are produced as a normal product of cellular metabolism. Specifically, one main cause of oxidative damage is hydrogen peroxide $\left(\mathrm{H}_{2} \mathrm{O}_{2}\right)$, which is 


\section{International Journal of Biochemistry \& Physiology}

converted from superoxide that leaks from the mitochondria. Catalase and superoxide dismutase ameliorate the damaging effects of hydrogen peroxide and superoxide, respectively, by converting these compounds into oxygen and hydrogen peroxide which is later converted to water, resulting in the production of benign molecules. However, this conversion is not $100 \%$ efficient, and residual peroxides persist in the cell. While ROS are produced as a product of normal cellular functioning, excessive amounts can cause damage [21]. Memory capabilities decline with age, evident in human degenerative diseases such as Alzheimer's disease, which is accompanied by an accumulation of oxidative damage. Current studies demonstrate that the accumulation of ROS can decrease an organism's fitness because oxidative damage is a contributor to senescence. In particular, the accumulation of oxidative damage may lead to cognitive dysfunction, as demonstrated in a study in which old rats were given mitochondrial metabolites and then given cognitive tests. Results showed that the rats performed better after receiving the metabolites, suggesting that the metabolites reduced oxidative damage and improved mitochondrial function [22]. Accumulating oxidative damage can then affect the efficiency of mitochondria and further increase the rate of ROS production [23]. This process continues till it reaches a damaging amounts which leads to mitochondrial DNA damage [24]. The accumulation of oxidative damage and its implications for aging depends on the particular tissue type where the damage is occurring. Additional experimental results suggest that oxidative damage is responsible for age-related decline in brain functioning. Older gerbils were found to have higher levels of oxidized protein in comparison to younger gerbils [25]. Treatment of old and young mice with a spin trapping compound caused a decrease in the level of oxidized proteins in older gerbils but did not have an effect on younger gerbils. In addition, older gerbils performed cognitive tasks better during treatment but ceased functional capacity when treatment was discontinued, causing oxidized protein levels to increase. This led researchers to conclude that oxidation of cellular proteins is potentially important for brain function [26].

In addition to Reactive Oxygen Species, due to high metabolic rate of the neurons and central nervous system, the amounts of Reactive Nitrogen Species (RNS) can go high as well. Reactive nitrogen species (RNS) are anti-microbial molecules derived from nitric oxide and superoxide produced through the enzymatic activity of nitric oxide synthase 2 (NOS2) and NADPH oxidase. Reactive nitrogen species act along with reactive oxygen species (ROS) to cause damage to the cells and also neurons, causing nitrosamine stress. Therefore, these two species are referred to as ROS/RNS [27].

\section{Lamarckian Evolutionary Hypothesis}

Lamarck stated the following two laws:

First Law: In every animal which has not passed the limit of its development, a more frequent and continuous use of any organ gradually strengthens, develops and enlarges that organ, and gives it a power proportional to the length of time it has been so used; while the permanent disuse of any organ imperceptibly weakens and deteriorates it, and progressively diminishes its functional capacity, until it finally disappears [28].

Second Law: All the acquisitions or losses wrought by nature on individuals, through the influence of the environment in which their race has long been placed, and hence through the influence of the predominant use or permanent disuse of any organ; all these are preserved by reproduction to the new individuals which arise, provided that the acquired modifications are common to both sexes, or at least to the individuals which produce the young [29].

In essence, a change in the environment brings about change in needs, resulting in change in behavior, bringing change in organ usage and development, bringing change in form over time-and thus the gradual transmutation of the species [30].

\section{Evolutionary Origin of Mitochondria}

Unlike any other organelle, except for chloroplasts, mitochondria appear to originate only from other mitochondria [31]. They contain their own DNA, which is circular as is true with bacteria, along with their own transcriptional and translational machinery. Mitochondrial ribosomes and transfer RNA molecules are similar to those of bacteria, as are components of their membrane. These and related observations led Dr. Lynn Margulis, in the 1970s, to propose an extracellular origin for mitochondria [32]. The endosymbiosis hypothesis for the origin of mitochondria (and chloroplasts) suggests that mitochondria are descended from specialized bacteria (probably purple non-sulfur bacteria) that somehow survived endocytosis by another species of prokaryote or some other cell type, and became incorporated into the cytoplasm. The ability of symbiont bacteria to conduct cellular respiration in host cells that relied on glycosis and fermentation would have provided a considerable evolutionary advantage. Similarly, host cells with symbiont bacteria capable of photosynthesis would also have an advantage. In both cases, the number of environments in which the cells could survive would have been greatly expanded [33].

Mitochondria do not contain anywhere near the amount of DNA needed to code for all mitochondriaspecific proteins, however, a billion or so years of 


\section{International Journal of Biochemistry \& Physiology}

evolution could account for a progressive loss of independence. The endosymbiosis hypothesis might be called a theory, but experimental evidence can't be provided to test it. Only circumstantial evidence is available in support of the proposal, which is the most likely explanation for the origin of mitochondria [34].

\section{Evolutionary Metabolic Hypothesis of Cancer}

The first living cells on Earth are thought to have arisen more than $3.5 \times 109$ years ago, when the Earth was not more than about 109 years old. The environment lacked oxygen but was presumably rich in geochemically produced organic molecules, and some of the earliest metabolic pathways for producing ATP may have resembled present-day forms of fermentation. In the process of fermentation, ATP is made by a phosphorylation event that harnesses the energy released when a hydrogen-rich organic molecule, such as glucose, is partly oxidized. The electrons lost from the oxidized organic molecules are transferred via NADH or NADPH to a different organic molecule or to a different part of the same molecule, which thereby becomes more reduced. At the end of the fermentation process, one or more of the organic molecules produced are excreted into the medium as metabolic waste products. Others, such as pyruvate, are retained by the cell for biosynthesis. The excreted end-products are different in different organisms, but they tend to be organic acids. Among the most important of such products in bacterial cells are lactic acid which also accumulates in anaerobic mammalian glycolysis, and formic, acetic, propionic, butyric, and succinic acids [35].

The first cell on the earth before the entrance of the bacteria did contain nucleus and used the fermentation process to produce ATP for its energy. Then an aerobic proteo-bacterium enters the eukaryote either as a prey or a parasite and manages to avoid digestion. It then became an endosymbiont. As we observe, the fermentation process used the glucose or even glutamine to produce ATP, but the aerobic process used the glucose, fat and protein to produce more ATP than the previous one. The symbio-genesis of the mitochondria is based on the natural selection of Charles Darwin. Based on Otto Warburg Hypothesis, in nearly all cancer cells, the mitochondrion is shut down or are defected and the cancer cell do not use its mitochondrion to produce ATP [36]. This process of adaptation is based on Lamarckian Hypothesis of Evolution and the normal cells goes back to the most primitive time of evolution to protect itself from apoptosis and uses the fermentation process like the first living cells 1.5 billion years ago. Therefore, cancer is an evolutionary metabolic disease which uses glucose as the main food to produce ATP and Lactic Acid. The prime cause of cancer is the abundance of Reactive Oxygen Species produced by mitochondria that is a threat to the living normal cell and causes mitochondrial damage mainly in its cristae [37].

\section{Relation between Cancer and Chaos Theory}

In animal cells, mitochondria are unique organelles in that they contain a genome of their own. There are small circular chromosomes in each mitochondrion that have genes for some of the mitochondrial proteins. But the mitochondrial chromosomes do not have genes for all the proteins found in mitochondria. The genes for the remaining proteins are found in the cellular genome which is found in the nucleus. So to get some functional mitochondria requires gene expression of both nuclear and mitochondrial genes. The human mitochondrial genome is a small circular DNA molecule 16568 bp in length containing 37 genes [38].

Twenty-four of the genes specify RNA molecules involved in protein synthesis while the remaining 13 encode proteins required for the biochemical reactions that make up respiration. The remaining mitochondrial OXPHOS proteins, the metabolic enzymes, the DNA and RNA polymerases, the ribosomal proteins and the mtDNA regulatory factors are all encoded by nuclear genes, synthesized in the cytosol and then imported intothe organelle. There is coordination of both nuclear and mitochondrial genes during mitochondrial function and biogenesis [39].

The main cause of cancer is the damage to the mitochondria in normal cells. Nearly all cancer cells contain damaged mitochondria and the real reason behind this is increasing inflammation or Reactive Oxygen Species produced by each mitochondrion. Increasing the ROS in a cell can cause damage to the mitochondrion DNA and also Nucleus DNA, but another reason behind turning the normal cell into cancer cell is the chaos caused by the increasing of ROS. These chaos causes some abnormal messaging between the DNA of the nucleus to stop the apoptosis and turning the oxidative phosphorylation to the fermentation in cytosol. Normally by damaging to the mitochondria, the cell should go to apoptosis estate, however; the nucleus sends wrong messages to stop the apoptosis and do fermentation process to survive the cell. Even some normal left mitochondria would be shut down and stop the oxidative phosphorylation. This is the main and the real reason why increasing intracellular inflammation can cause cancer. [Somayeh Zaminpira, Sorush Niknamian, ECRONICON, 2017]

The prime cause of cancer is the damage to the mitochondria in normal cells. Nearly all cancer cells contain damaged mitochondria and the basic reason behind this, is increasing the intracellular inflammation or basically the incline in Reactive Oxygen Species (ROS) produced by each mitochondrion in oxidative phosphorylation. Increasing the ROS in a cell can cause 


\section{International Journal of Biochemistry \& Physiology}

damage to the DNA of the mitochondrion and also Nucleus DNA, but another reason behind turning the normal cell into cancer cell is the chaos caused by the increasing of inflammation inside each cell and increasing the intracellular ROS [40]. These chaos causes some abnormal messaging between the DNA of the nucleus to stop the apoptosis and turning the oxidative phosphorylation to the fermentation in cytosol. Normally by damaging to the mitochondria, the cell should apoptosis. however; the nucleus sends wrong messages to stop the apoptosis and do fermentation process in cytosol to survive the cell. Even some normal left mitochondria would be shut down and stop the oxidative phosphorylation. This is the main and the real reason how increasing intracellular inflammation can cause cancer. This research introduces the butterfly effect inside the normal cells is the basic reason behind the cause of cancer [41].

\section{Materials and Methods}

This research is set to find the differences between the normal eukaryote cells and different cancer cells. Additionally, the second aim of the research is the relation between the cancer cells and the primitive eukaryote cells in 3.5 billion years ago [42].

The research is done at the Violet Cancer Institute (VCI) with the supervision of Somayeh Zaminpira Ph.D. and Sorush Niknamian Ph.D.

We had collected 34 samples of different cancer tumor cells.

4 colorectal cancer tumors, 2 stomach cancer tumors, 5 liver cancer tumors, 5 brain cancer tumors, 5 breast cancer tumors, 3 prostatic cancer tumors, 2 bone cancer tumors, 1 lymphatic cancer tumor, 2 melanoma cancer samples and 5 lung cancer tumors.

All the experiments were done by the Real-time PCR device and electron microscopes.

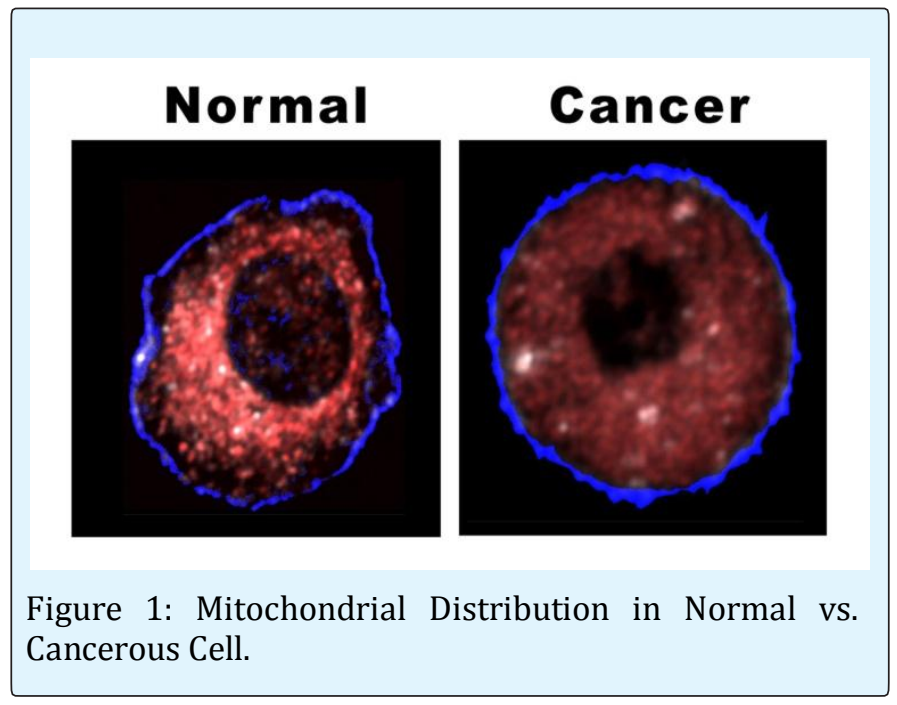

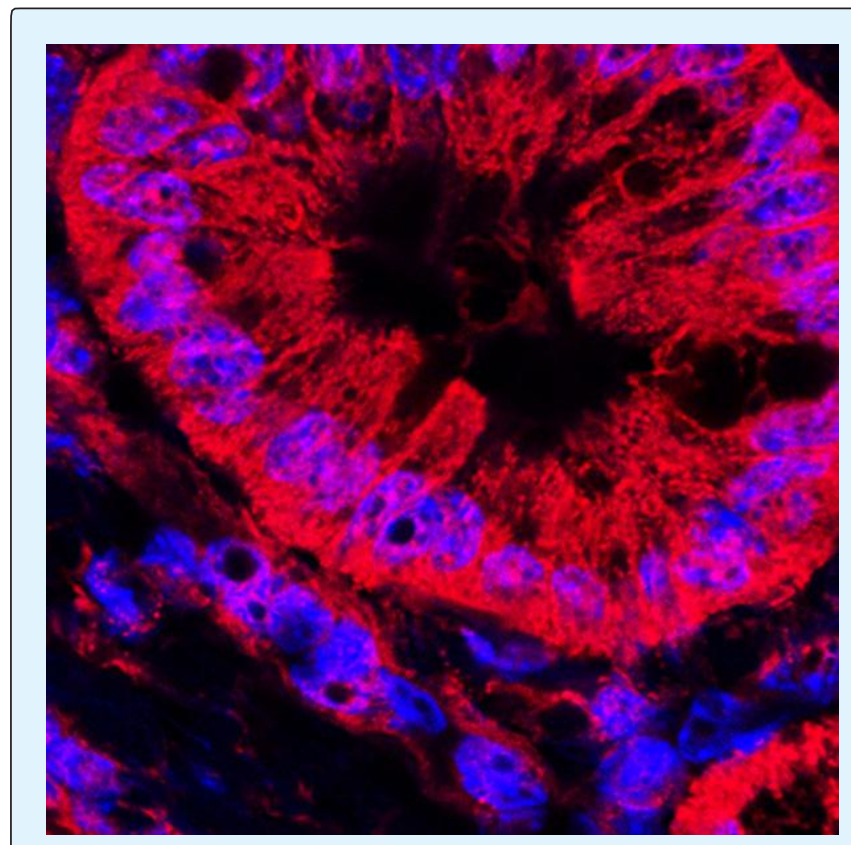

Figure 2: Mitochondrial Dynamics in Cancerous Cell. The cristae of the mitochondrion are damaged.

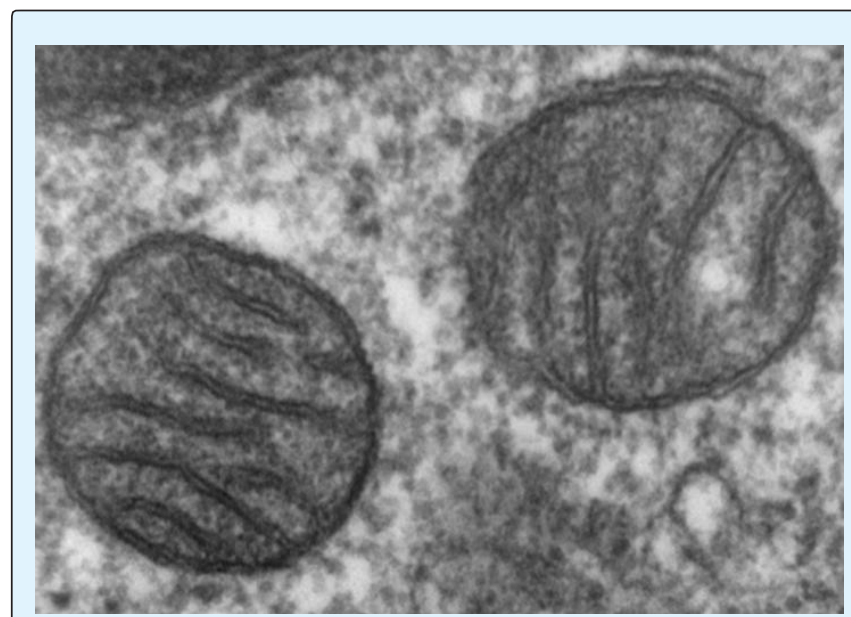

Figure 3: Mitochondria in cancer cell.

\section{Discussions and Results}

A) The results show that 64 percent of the mitochondria inside the cancer cells were damaged and most of them have occurred in their cristae. The remained 46 percent did not work or better said were shut down without any living signs.

B) All the cancer tumor cells used fermentation respiration and produced lactic acid. Even in the 99\% pure oxygen we did not observe any change in the respiration.

C) All the cancer tumor cells went through apoptosis state after they faced 5 percent ozone which was a wonderful result and showed the ozone causes the incline in the amounts of ROS in the cancer cells which made them go through apoptosis. 


\section{International Journal of Biochemistry \& Physiology}

\section{Introduction to Evolutionary Cell Memory Hypothesis (ECM)}

According to Evolutionary Metabolic Hypothesis of Cancer (EMHC) mentioned by S. Zaminpira and S. Niknamian in 2017, when the amount of ROS and RNS inside the normal eukaryote cells go beyond the safety limits and according to Otto Warburg, when the amount of oxygen goes down nearly under $30 \%$ of normal oxygen in tissue cells and the glucose amounts in blood are enough, normal cells become cancer cells which uses fermentation respiration.

Shifting the respiration mechanism in eukaryotic cells which includes mitochondrion metabolism raises a provocative thought. As nearly all scientists agree with the endosymbiosis theory of mitochondrion, these energy producing machines in human cells were a form of bacterium in earlier life. Therefore, going through all phases of this perspective research article, I propose a hypothesis called The Evolutionary Cell Memory (ECM) hypothesis which explains these types and changes in human cells to cancer cells.

Based on ECM hypothesis, mitochondrion in eukaryotic cells does have their primitive genes in earlier life and these genes explain the weird shift from normal cell into cancer cell based on Warburg Effect.

ECM hypothesis says that: "When the habitat of a microorganism which includes mitochondrion changes dramatically, the mitochondrion metabolism (or respiration process) changes to the way it was in the earlier simple life form (bacterium in water world theory) to saving its life from distinction."

In human cells, when the amount of oxygen goes down under $30 \%$ of normal oxygen needed for the cell to respire normally, mitochondria become damaged or shut down by the wrong order from the nucleus and the cells go from the aerobic estate of respiration to anaerobic fermentation estate in order to save their lives and continue the energy production for the tissue. This shift is amazing due to the ability of the normal eukaryote cell to change its shape into the earlier form in evolution. By this sudden shift, the cell will not distinct and multiply faster just like bacterium in the earlier form of life.

\section{Conclusion}

Nearly all the 34 samples of the cancer cells had damaged or shut down mitochondria. Nearly 3.5 billion years ago before the entrance of the mitochondria inside the eukaryote cells, the living cells did fermentation process without the usage of oxygen. If the amounts of the ROS or RNS inside the cells go beyond the safety limits, the normal cells shut down their mitochondria instead of going into apoptosis state to save themselves from dying. The quick multiplying and the structure of cancer cells has the closest resemblance to the primitive cells. This process made us introduce a new hypothesis which is called Evolutionary Cell Memory Hypothesis (ECM). It says: "When the habitat of a microorganism which includes mitochondrion changes dramatically, the mitochondrion metabolism (or respiration process) changes to the way it was in the earlier simple life form (bacterium in water world theory) to saving its life from distinction."

\section{References}

1. Longo VD, Fontana L (2010) Calorie restriction and cancer prevention: metabolic and molecular mechanisms. Trends Pharmacol Sci 31(2): 89-98.

2. World Health Organization (2017) "Cancer Fact Sheet N²97".

3. National Cancer Institute (2014) Defining Cancer.

4. NHS Choices (2014) Cancer - Signs and symptoms.

5. Seyfried TN, Flores RE, Poff AM, D'Agostino DP (2014) Cancer as a metabolic disease: implications for novel therapeutics. Carcinogenesis 35(3): $515-$ 527.

6. Biffi, A Montini E, Lorioli L, Cesani M, Fumagalli F, et al. (2013) Lentiviral Hematopoietic Stem Cell Gene Therapy Benefits Metachromatic Leukodystrophy. Science 341(6148): 1233158.

7. Parkin, DM; Boyd, L; Walker, LC (2011) 16. The fraction of cancer attributable to lifestyle and environmental factors in the UK in 2010. Br J Cancer 105(2): S77-S81.

8. Pezzoli D1, Chiesa R, De Nardo L, Candiani G (2012) We still have a long way to go to effectively deliver genes. J Appl Biomater Funct Mater 10(2): 82-91.

9. Aiuti A Biasco L, Scaramuzza S, Ferrua F, Cicalese MP, et al. (2013) Lentiviral Hematopoietic Stem Cell Gene Therapy in Patients with Wiskott-Aldrich Syndrome. Science 341(6148): 1233151.

10. Whyte DA, Al-Hammadi S, Balhaj G, Brown OM, Penefsky HS, et al. (2010) Cannabinoids inhibit cellular respiration of human oral cancer cells. Pharmacology 85(6): 328-335.

11. Kaambre T, Chekulayev V, Shevchuk I, Tepp K, Timohhina N, et al. (2013) Metabolic control analysis of respiration in human cancer tissue. Front Physiol 4: 151. 


\section{International Journal of Biochemistry \& Physiology}

12. Gallagher James (2015) Gene therapy: 'Tame HIV' used to cure disease BBC News, Health.

13. Malech HL, Ochs HD (2015) An Emerging Era of Clinical Benefit from Gene Therapy. JAMA 313(15): 522-523.

14. Lee TW, Southern KW, Perry LA, Penny-Dimri JC, Aslam AA (2007) Topical cystic fibrosis transmembrane conductance regulator gene replacement for cystic fibrosis-related lung disease. Cochrane Database Syst Rev (2): CD005599.

15. MacLaren RE, Groppe M, Barnard AR, Cottriall CL, Tolmachova T, et al. (2014) Retinal gene therapy in patients with choroideremia: Initial findings from a phase 1/2 clinical trial. Lancet 383(9923): 11291137.

16. Tebas P, Stein D, Tang WW, Frank I, Wang SQ, et al. (2014) Gene Editing of CCR5in Autologous CD4 T Cells of Persons Infected with HIV". New England Journal of Medicine. 370(10): 901-910.

17. National Heart, Lung, and Blood Institute (2017) Collection and Storage of Umbilical Cord Stem Cells for Treatment of Sickle Cell Disease. ClinicalTrials.gov.

18. Gardner MR, Kattenhorn LM, Kondur HR, von Schaewen M, Dorfman T, et al. (2015) AAVexpressed eCD4-Ig provides durable protection from multiple SHIV challenges". Nature 519(7541): 87-91.

19. Wade Nicholas (2015) Scientists Seek Ban on Method of Editing the Human Genome. New York Times.

20. Rogers S (1970) New Scientist, pp: 194.

21. Vander Heiden MG, Cantley LC, Thompson CB (2009) Understanding the Warburg Effect: The Metabolic Requirements of Cell Proliferation. Science 324(5930): 1029-1033.

22. Deberardinis RJ, Lum JJ, Hatzivassiliou G, Thompson CB (2008) The biology of cancer: metabolic reprogramming fuels cell growth and proliferation. Cell Metab 7(1): 11-20.

23. Weinhouse S (1976) The Warburg hypothesis fifty years later. Z Krebsforsch Klin Onkol Cancer Res Clin Oncol 87(2): 115-126.

24. Shaw RJ, Kosmatka M, Bardeesy N, Hurley RL, Witters LA, et al. (2004) The tumor suppressor LKB1 kinase directly activates AMP-activated kinase and regulates apoptosis in response to energy stress. Proc Natl Acad Sci USA 101(10): 3329-3335.

25. Christofk HR, Vander Heiden MG, Wu N, Asara JM, Cantley LC (2008) Pyruvate kinase M2 is a phosphotyrosine-binding protein. Nature 452(7184): 181-186.

26. Wolf U (1974) Theodor Boveri and his book, on the problem of the origin of malignant tumors. In German J (Ed), Chromosomes and Cancer, John Wiley \& Sons, New York, pp: 1-20.

27. Cairns J (1981) The origin of human cancers. Nature 289(5796): 353-357.

28. Steele EJ (2016) Somatic hypermutation in immunity and cancer: Critical analysis of strandbiased and codon-context mutation signatures. DNA Repair 45: 1-24.

29. Steele EJ (1981) Somatic selection and adaptive evolution: on the inheritance of acquired characters $2^{\text {nd }}$ (Edn.), Chicago: University of Chicago Press.

30. Roth TL, Lubin FD, Funk AJ, Sweatt JD (2009) Lasting Epigenetic Influence of Early-Life Adversity on the BDNF Gene". Biol Psychiatry 65(9): 760-769.

31. Arai JA, Li S, Hartley DM, Feig LA (2009) Transgenerational Rescue of a Genetic Defect in Long-Term Potentiation and Memory Formation by Juvenile Enrichment. J Neurosci 29(5): 1496-1502.

32. Hackett JA, Sengupta R, Zylicz JJ, Murakami K, Lee C, et al. (2013) Germline DNA Demethylation Dynamics and Imprint Erasure Through 5Hydroxymethylcytosine. Science. 339(6118): 448452.

33. Bonduriansky R (2012) Rethinking heredity, again. Trends Ecol Evol 27(6): 330-336.

34. Skinner MK (2015) Environmental Epigenetics and a Unified Theory of the Molecular Aspects of Evolution: A Neo-Lamarckian Concept that Facilitates Neo-Darwinian Evolution. Genome Biol Evol 7(5): 1296-1302.

35. Loeb LA (2001) A mutator phenotype in cancer. Cancer Res. 61(8): 3230-3239.

36. Wagner RP (1999) Anecdotal, historical and critical commentaries on genetics. Rudolph Virchow and the genetic basis of somatic ecology. Genetics 151(3): 917-920.

37. Howell AN, Sager R (1978) Tumorigenicity and its suppression in cybrids of mouse and Chinese 


\section{International Journal of Biochemistry \& Physiology}

hamster cell lines. Proc. Natl Acad Sci USA 75(5): 2358-2362.

38. Jonasson J, Harris H (1977) The analysis of malignancy by cell fusion. VIII. Evidence for the intervention of an extra-chromosomal element. J Cell Sci 24: 255-263.

39. Seyfried TN (2012) Mitochondria: the ultimate tumor suppressor. In: Cancer as a Metabolic Disease: On the Origin, Management, and Prevention of Cancer. John Wiley \& Sons, Hoboken, NJ, pp: 195-205.
40. Kaipparettu BA, Ma Y, Park JH, Lee TL, Zhang Y, et al. (2013) Crosstalk from non-cancerous mitochondria can inhibit tumor properties of metastatic cells by suppressing oncogenic pathways. PLoS One 8(5): e61747.

41. Israel BA, Schaeffer WI (1988) Cytoplasmic mediation of malignancy. In Vitro Cell Dev Biol 24(5): 487-490.

42. Gregory TR (2009) Lamarck didn't say it, Darwin did". Genomicron. 